\title{
Application of numerical method to investigation of fatigue crack behavior through the friction stir welding
}

\begin{abstract}
Fatigue crack propagation through a friction stir welded (FSW) joint of 2024-T351 Al alloy is investigated numerically. The governing relationships for predicting the crack behavior including incremental crack length, crack growth rate, and crack growth direction are presented. Stress intensity is calculated based on displacement correlation technique, and fatigue crack growth through the FSW joint is investigated under linear elastic fracture mechanics (LEFM) using the Paris model. The concepts of crack closure, residual stress, and stress relaxation are incorporated into the Paris model to support the final results. Maximum circumferential tensile stress method is applied to predict the crack growth direction. Finally, the numerical approaches are employed to the high number of elements in the framework of Fracture Analysis Code (FRANC2D/L) to simulate the fatigue crack propagation through the FSW joint including various zones with different material properties. Fatigue lifetime of the welded joint is predicted by implementing the same procedure for various loading values. The obtained numerical results are validated with the experimental work (Ali et al., Int $\mathbf{J}$ Fatigue 30:2030-2043, 2008).
\end{abstract}

Keyword: Fatigue crack growth; Finite element method; Friction stir welding; Numerical analysis; Simulation 